\title{
Effect of Nocturnal Oxygen on Blood Pressure Response to Altitude Exposure in COPD - Data from a Randomized Placebo-Controlled Cross- Over Trial
}

\author{
Martina Meszaros' \\ Tsogyal D Latshang' \\ Sayaka S Aeschbacher' \\ Fabienne Huber \\ Deborah Flueck' \\ Mona Lichtblau' \\ Stefanie Ulrich' \\ Elisabeth D Hasler' \\ Philipp M Scheiwiller ${ }^{\prime}$ \\ Lukas Reinhard' \\ Silvia Ulrich (D) $^{1,2}$ \\ Konrad E Bloch ${ }^{1-3}$ \\ Michael Furian (D) \\ Esther I Schwarz $\mathbb{D}^{1,3}$ \\ 'Department of Pulmonology and Sleep \\ Disorders Centre, University Hospital \\ Zurich, Zurich, Switzerland; ${ }^{2}$ Zurich \\ Center for Integrative Human Physiology \\ (ZIHP), University of Zurich, Zurich, \\ Switzerland; ${ }^{3}$ Centre of Competence \\ Sleep \& Health, University of Zurich, \\ Zurich, Switzerland
}

Correspondence: Esther I Schwarz Department of Pulmonology and Sleep Disorders Centre, University Hospital Zurich, Zurich, Switzerland

Tel +4I 44255 II II

$\mathrm{Fax}+4$ I 44255445 I

Email estherirene.schwarz@usz.ch
Purpose: Patients with chronic obstructive pulmonary disease (COPD) are particularly vulnerable to hypoxia-induced autonomic dysregulation. Hypoxemia is marked during sleep. In COPD, altitude exposure is associated with an increase in blood pressure (BP) and a decrease in baroreflex-sensitivity (BRS). Whether nocturnal oxygen therapy (NOT) may mitigate these cardiovascular autonomic changes in COPD at altitude is unknown.

Materials and Methods: In a randomized placebo-controlled cross-over trial, 32 patients with moderate-to-severe COPD living $<800 \mathrm{~m}$ were subsequently allocated to NOT and placebo during acute exposure to altitude. Measurements were done at low altitude at $490 \mathrm{~m}$ and during two stays at $2048 \mathrm{~m}$ on NOT $(3 \mathrm{~L} / \mathrm{min})$ and placebo $(3 \mathrm{~L} / \mathrm{min}$, ambient air) via nasal cannula. Allocation and intervention sequences were randomized. Outcomes of interest were BP, BRS (from beat-to-beat BP measurement), BP variability (BPV), and heart rate.

Results: About 23/32 patients finished the trial per protocol (mean (SD) age 66 (5) y, $\mathrm{FEV}_{1}$ 62 (14) \% predicted) and 9/32 experienced altitude-related illnesses ( 8 vs $1, p<0.05$ placebo vs NOT). NOT significantly mitigated the altitude-induced increase in systolic BP compared to placebo $(\Delta$ median $-5.8[95 \% \mathrm{CI}-22.2$ to -1.4$] \mathrm{mmHg}, \mathrm{p}=0.05)$ but not diastolic BP $(-3.5$ [95\% CI -12.6 to 3.0$] \mathrm{mmHg} ; \mathrm{p}=0.21)$ or BPV. BRS at altitude was significantly higher in NOT than in placebo $(1.7$ [95\% CI 0.3 to 3.4$] \mathrm{ms} / \mathrm{mmHg}, \mathrm{p}=0.02)$.

Conclusion: NOT may protect from hypoxia-induced autonomic dysregulation upon altitude exposure in COPD and thus protect from a relevant increase in BP and decrease in BRS. NOT may provide cardiovascular benefits in COPD during conditions of increased hypoxemia and may be considered in COPD travelling to altitude.

Keywords: COPD, altitude, oxygen, hypoxia, blood pressure, blood pressure variability, baroreflex sensitivity

\section{Introduction}

Chronic obstructive pulmonary disease (COPD) is common - about $10 \%$ of the adult population is affected. ${ }^{1}$ COPD is characterized by irreversible destruction of the small airways leading to airflow limitation, hyperinflation and impairment in gas exchange. COPD is one of the leading causes of death worldwide and is frequently associated with cardiovascular comorbidities, such as hypertension, coronary heart disease and right ventricular dysfunction. ${ }^{2}$ Air traveling and altitude 
stays are usual practices in modern society. These activities are exposing patients with COPD to hypoxia and result in marked hypoxemia. Other conditions of marked hypoxemia in patients with COPD are acute exacerbations of COPD and lower respiratory tract infections or decompensated heart failure. Hypoxemia triggers autonomic cardiovascular dysregulation and has been shown to result in increased sympathetic activity by chemoreflex activation. ${ }^{3}$ Baroreflex sensitivity (BRS) is a key player in autonomic regulation of the cardiovascular system and also relevant for rapid adjustment of cardiac output. ${ }^{4}$ Baroreceptors are localized in the aortic arch and carotid sinus and are activated by stretching of arterial walls as blood pressure (BP) increases. ${ }^{5}$ Autonomic dysregulation and altered BRS are present in a wide range of cardiovascular disease and have been postulated to be a risk factor for sudden cardiac death. $^{6-8}$ Sympathetic overactivity plays a role in COPD and its degree seems to be predictive of hospitalizations and mortality. ${ }^{9}$ The consequential autonomic dysregulation and impaired baroreflex lead to increased cardiovascular morbidity and mortality in patients with COPD. ${ }^{10-12}$ It was hypothesized that pulmonary hypertension (WHO group 3) is an independent predictor of attenuated BRS in COPD and it was shown that the attenuation of BRS was more pronounced in subjects with COPD and a lower arterial partial pressure of oxygen $\left(\mathrm{pO}_{2}\right){ }^{13}$

We have previously shown that exposure to high altitude and associated hypobaric hypoxia results in a blunted BRS in COPD. ${ }^{14}$ Compared to sea level, patients with COPD showed more pronounced hypoxemia, decreased exercise capacity, impaired quality of life and increased BP at altitude. ${ }^{15-18}$ Upon exposure to hypoxia at altitude, stimulation of the peripheral chemoreceptors and other mechanisms lead to sympathetic activation and a blunted baroreflex. ${ }^{19,20}$ We have previously shown that BP significantly increases when patients with moderate or severe COPD are exposed to hypobaric hypoxia at altitude. ${ }^{14}$ This BP increase was accompanied by an increase in blood pressure variability (BPV) and a decrease in BRS as indicator of a disturbed autonomic cardiovascular regulation. ${ }^{14}$ The respiratory and cardiovascular changes experienced at altitude must be taken into consideration in patients with COPD. Several rehabilitation clinics are located at moderate altitude and patients with COPD stay at altitude for recreational activities, such as hiking or sports and might therefore be more physically active and exercise there. Moreover, commercial air flights may also be problematic for patients with COPD due to exposure to hypoxia.

Supplemental oxygen therapy has been shown to reduce dyspnea, improve exercise performance and reduce mortality in severe resting hypoxemia, whereas the evidence for isolated nocturnal hypoxemia is less clear. ${ }^{21-26}$

We hypothesized that nocturnal oxygen therapy (NOT) will attenuate the pronounced increase in BP and impairment of BRS previously observed in patients with COPD exposed to moderate altitude. Therefore, the aim of this randomized controlled interventional trial was to evaluate whether NOT may prevent these adverse effects on BP and BRS in COPD during exposure to altitude.

\section{Materials and Methods Study Design, Intervention and Randomization}

In a randomized, single-blind, placebo-controlled crossover trial, patients were studied in random order at low altitude (490 m, Zurich, Switzerland) breathing room air, once during a two-day stay at moderate altitude $(2048 \mathrm{~m}$, St. Moritz, Switzerland) using nocturnal oxygen therapy (NOT), and once during another two-day stay at moderate altitude using nocturnal placebo (room air). There was a minimal interval of two-weeks at $<800 \mathrm{~m}$ in-between each study visit. Both allocation and intervention sequences were randomized. At altitude, participants received NOT $(3 \mathrm{~L} / \mathrm{min})$ or placebo $(3 \mathrm{~L} / \mathrm{min}$ room air) through a nasal cannula connected to a concentrator (EverFlow, Philips Respironics, Murrysville, PA, USA). Blinding of participants was achieved by placing the concentrator in a separate room. Due to safety considerations and nocturnal monitoring of all participants, blinding of the study investigators was not feasible. For details on randomization, we refer to a previously published study protocol and published findings related to sleep and sleepdisordered breathing. ${ }^{26}$ Results related to BP and autonomic regulation have not been published previously.

All procedures were performed in accordance with the ethical standards of the institutional and national research committee and with the 1964 Helsinki declaration. The study protocol has been approved by the Ethics Committee of the Canton of Zurich (EK-2013-0088) and registered at ClinicalTrials.gov (registration number NCT02150590). Written informed consent was obtained from each participant. 


\section{Participants}

Men and women, age 18-75 years with COPD, GOLD (Global Initiative for Chronic Obstructive Lung Disease) grades 2 to $3\left(\mathrm{FEV}_{1} / \mathrm{FVC}<0.7\right.$ and $\mathrm{FEV}_{1} 30-80 \%$ predicted), living at low altitude $(<800 \mathrm{~m})$ were eligible. Patients with hypoxemia at low altitude $\left(\mathrm{SpO}_{2}<92 \%\right.$ at $490 \mathrm{~m}$ ), using oxygen supplementation or positive pressure ventilation therapy, with unstable COPD, heavy smokers ( $>20$ cigarettes/day), with severe or uncontrolled cardiovascular disease, previous intolerance of altitude exposure, or an altitude stay $(>1500 \mathrm{~m})$ within the last four weeks were excluded.

\section{Outcomes and Assessments}

The main outcomes of interest were differences in the change in the morning BP, BRS, heart rate, and BPV expressed as coefficients of variation of systolic and diastolic BP between NOT and placebo at high altitude $(2048 \mathrm{~m})$ compared to low altitude $(490 \mathrm{~m})$. All the measurements were carried out in the morning under standardized environmental circumstances (supine position after rest for $>10$ minutes in a quiet room with stable temperature and lightning) at both altitudes. Morning systolic and diastolic BP and heart rate were measured by the volume-clamp method using a Finometer $^{\circledR}$ device (Finapres Medical System, Amsterdam, the Netherlands) and with the conventional Riva-Rocca method. Beat-tobeat BP and heart rate were measured continuously for at least 6 minutes by the Finometer ${ }^{\circledR}$ device. BRS was calculated using the Beatscope ${ }^{\circledR}$ Software (Version 1.1.a., Finapres Medical System, Amsterdam, the Netherlands) using the cross-correlation sequence method and expressed in $\mathrm{msec} / \mathrm{mmHg}{ }^{27}$

In addition, polysomnography, arterial blood gas analysis, lung function testing and 6-minute walk testing (6-MWT) were performed as previously reported. ${ }^{26}$

The sample size was calculated to detect a difference in mean (SD) $\mathrm{SpO} 2$ of $2 \%(4 \%)$ between NOT and placebo with an $\alpha$ error probability of 0.05 and a power of $0.80 .{ }^{26}$

\section{Statistical Analysis}

Statistical analyses were performed with IBM SPSS (SPSS Inc., Chicago, USA) and Graph Pad Prism 5.0 (GraphPad Software, San Diego, CA, USA). The normality of the data was tested using Shapiro-Wilk test and Kolmogorov-Smirnov test. Normally distributed data were expressed as mean with standard deviation (SD) and non-normally distributed data were expressed as median with interquartile range (IQR, 25-75 percentile). Since the outcomes of interest were secondary outcomes in the protocol and physiological measures, a per-protocolanalysis was performed to assess the effect of altitude exposure and NOT on BP, BRS and HR. Changes in the variables between the sojourns were assessed by ANOVA for repeated measurements with Fisher post hoc analysis or by Friedman test (non-parametric alternative to the oneway ANOVA with repeated measures) with Conover's post hoc pairwise comparisons based on normality testing. A $p$ value $<0.05$ was considered significant.

\section{Results}

\section{Study Flow and Patient Characteristics}

The trial ran from June 12014 to October 31 2014. Out of 32 patients randomized, 23 finished the trial per protocol and received the allocated intervention during both altitude sojourns (Figure 1). Eight patients under placebo and 1 patient under NOT ( $\mathrm{P}<0.05$ between treatments) did not receive the allocated intervention due to predefined safety precautions due to severe nocturnal hypoxemia requiring oxygen therapy ( $\mathrm{n}=4, \mathrm{SpO}_{2}<75 \%$ for $>30 \mathrm{~min}$ ), COPD exacerbation $(n=2)$, newly onset cardiovascular disease (arterial hypertension or coronary heart disease; $\mathrm{n}=2$ ), or panic attack $(n=1)$. The per protocol analysis included 23 patients. The 9 patients who were not included in the per protocol analysis did not differ in their baseline characteristics from the per protocol population (see Supplementary Table 1). Data on BRS were only available from 12 patients due to artifacts in the beat-to-beat-BPmeasurement in at least one of the three measurements. The characteristics of the participants are presented in Table 1. Previously reported data from polysomnographies are reported in Supplementary Table 2.

\section{Effect of Altitude Exposure on BP, BRS, Heart Rate and BPV}

Altitude exposure resulted in an increase in systolic and diastolic blood pressure and heart rate as well as a reduction in BRS, while BPV was not affected (Table 2, Figure 2).

\section{Treatment Effect of NOT on BP, BRS, Heart Rate and BPV}

NOT abolished the increase in systolic BP upon altitude exposure. NOT resulted in a significant reduction in 


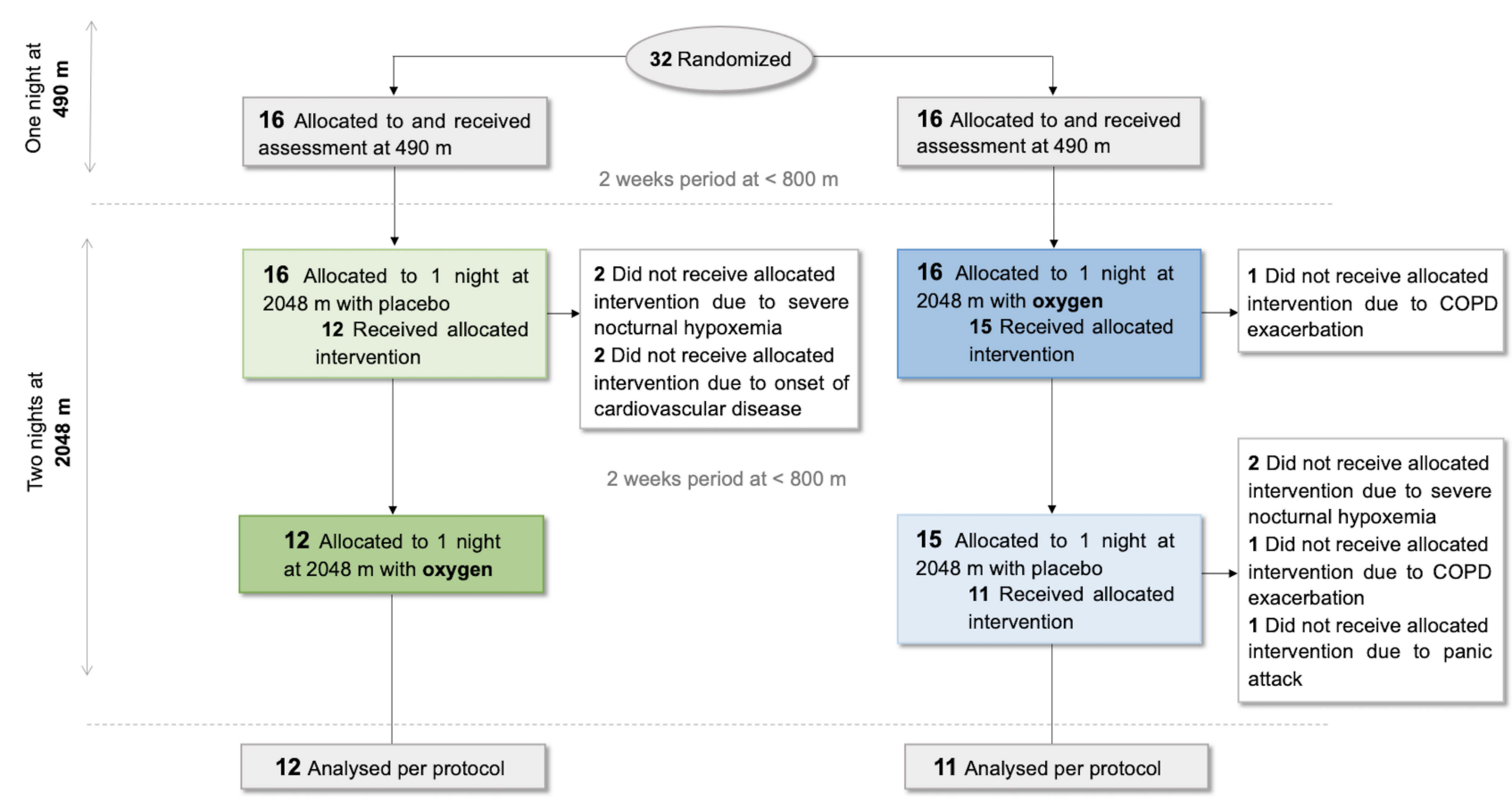

Figure I Simplified patient flow chart. See ${ }^{26}$ for four arm allocation of the intention-to-treat-analysis in the main trial. Severe hypoxemia was defined as an arterial oxygenation measured by finger oximetry $<75 \%$ for $>30 \mathrm{~min}$.

systolic morning BP at altitude compared to placebo (Table 2, Figure 2). However, there was no significant difference in the morning diastolic BP between placebo and NOT at altitude (Table 2, Figure 1). BRS at altitude was significantly higher when using NOT compared to placebo. NOT prevented the altitude-induced reduction in BRS (Table 2, Figure 1). Heart rate was significantly lower at altitude when using NOT compared to placebo (Table 2, Figure 1). NOT had no effect on variability of systolic or diastolic BP (BPV) compared to placebo (Table 2).

\section{Discussion}

This randomized placebo-controlled trial has shown that acute altitude exposure increases systolic and diastolic blood pressure and heart rate in patients with moderate and severe COPD. NOT reduced the increase in systolic $\mathrm{BP}$ and heart rate and improved BRS at altitude. These findings imply that NOT reduces hypoxia-induced sympathetic activation and autonomic dysregulation. This may be important both in terms of exercise capacity and cardiovascular complications for COPD patients during altitude stays or air travel. These findings are applicable to moderate-to-severe COPD not being hypoxic at low altitude. Application of NOT is practicable and does not interfere with daytime activities.
Previous studies provided insight into mechanisms involved in hypoxia-induced autonomic dysregulation in COPD. There is an inhibitory relationship between baroand chemoreflexes, thus the increased activity of the peripheral chemoreceptors induced by hypoxia contributes to reduced BRS. ${ }^{19}$ The inhibition of chemoreceptors attenuates the decrease in BRS under hypoxic conditions. ${ }^{28}$ Hypobaric hypoxia at altitude has been shown to reset the set point of baroreflex towards higher BP and to lower the reflex gain resulting in autonomic dysregulation of BP. ${ }^{29,30}$ It was demonstrated in healthy participants at $5260 \mathrm{~m}$ that acute hypoxia causes a decrease in BRS, which was exacerbated by longer exposure to hypoxia after 16 days. ${ }^{31}$ Acute hypoxia-induced hyperventilation also impairs the baroreflex gain by causing hypocapnia and this decrease in BRS is shown to be reversible after inspiration of carbon dioxide $\left(\mathrm{CO}_{2}\right){ }^{31,32}$ The authors suggested that sustained activation of $\mathrm{CO}_{2}$ chemoreceptors could be one of the factors causing blunted BRS associated with acute hypoxia at high altitude and increased sensitivity of chemoreceptors may worsen BRS during chronic hypoxia. ${ }^{31}$ Interestingly, a recent study revealed that baroreceptors located in pulmonary arteries may also regulate sympathetic outflow contributing to the resetting of baroreflex at high altitude. Patients with COPD may have pulmonary hypertension and its aggravation by 
Table I Patient Characteristics in the per Protocol Population

\begin{tabular}{|c|c|}
\hline Variable & $\mathbf{N}=\mathbf{2 3}$ \\
\hline Men, No (\%) & II (48) \\
\hline Women, No (\%) & $12(52)$ \\
\hline Age, years & $66.0(5.3)$ \\
\hline Body Mass Index, $\mathrm{kg} / \mathrm{m}^{2}$ & $25.1(3.7)$ \\
\hline $\mathrm{FEV}_{1}$, liters & $1.7(0.5)$ \\
\hline $\mathrm{FEV}_{\mathrm{I}}$ \% predicted & $62(14)$ \\
\hline FVC, liters & $3.2(0.7)$ \\
\hline FVC, \% predicted & $90(12)$ \\
\hline $\mathrm{FEV}_{1 /} \mathrm{FVC}$, absolute value & $0.5(0.1)$ \\
\hline $\mathrm{TL}_{\mathrm{CO}}(\mathrm{Hb}), \mathrm{mmol} / \mathrm{min} / \mathrm{kPa}$ & $5.7(2.0)$ \\
\hline $\mathrm{TL}_{\mathrm{CO}}(\mathrm{Hb}), \%$ predicted & $70(20)$ \\
\hline GOLD grade 2, No (\%) & $18(78)$ \\
\hline \multicolumn{2}{|l|}{ Comorbidities } \\
\hline $\begin{array}{l}\text { Cardiovascular disease (hypertension or coronary heart } \\
\text { disease), No. (\%) }\end{array}$ & $14(60)$ \\
\hline Diabetes, No (\%) & $3(13)$ \\
\hline Depression, No (\%) & $3(13)$ \\
\hline \multicolumn{2}{|l|}{ Medication } \\
\hline Inhaled glucocorticoids, No (\%) & $9(39)$ \\
\hline Inhaled $\beta$-adrenergics, No (\%) & $16(70)$ \\
\hline Inhaled anticholinergics, No (\%) & $16(70)$ \\
\hline Diuretics, No (\%) & $4(17)$ \\
\hline Antihypertensive medication, No (\%) & II (48) \\
\hline -Beta-blocker, No (\%) & I (4) \\
\hline -ACE-I or ARB, No (\%) & $9(39)$ \\
\hline -Calcium channel blocker, No (\%) & $6(26)$ \\
\hline -Diuretics and other antihypertensive medications, No (\%) & $5(22)$ \\
\hline Antidiabetics, No (\%) & $3(13)$ \\
\hline Antidepressants, No (\%) & $5(22)$ \\
\hline
\end{tabular}

Notes: Continuous variables are presented as mean (SD) and categorical variables are presented as numbers (\%).

Abbreviations: $A C E l$, angiotensin-converting enzyme inhibitors; $A R B$, angiotensin receptor blockers; $\mathrm{FEV}_{\mathrm{l}}$, forced expiratory volume in the first second of expiration; FVC, forced vital capacity; TLco, transfer factor of the lung for carbon monoxide; GOLD, Global Obstructive Lung Disease classification.

hypobaric hypoxia may affect autonomic cardiovascular regulation via pulmonary arterial baroreceptors. ${ }^{33}$ Hypoxia-induced activation of the renin-angiotensinsystem (RAS) may be involved in impaired baroreflex in COPD. ${ }^{34}$ Angiotensin II also modulates neurotransmitters in vagal efferents and acts on firing of the peripheral baroreceptors, ${ }^{35,36}$ thus may possibly alter baroreflex in COPD.

Sustained sympathetic activation due to hypoxia plays an important role in impaired autonomic regulation in COPD. ${ }^{9}$ Increased sympathetic activity has been considered to contribute to arterial thickening and increased arterial stiffness, ${ }^{37}$ which is an independent risk factor for cardiovascular disease and is associated with emphysema severity in patients with COPD. ${ }^{38}$ The stiffness in elastic arteries causes the deformation of the baroreceptors leading to decreased BRS and its impaired efficacy to buffer BP fluctuations. ${ }^{39}$ It is worth noting that sleep-related breathing disorders frequently associated with COPD (for example, obstructive sleep apnoea) are partly responsible for the worsening of autonomic control by nocturnal intermittent hypoxia and sympathetic activation. ${ }^{40}$

Favorable cardiovascular effects of long-term oxygen therapy in COPD have been shown in different studies as hypoxia is known to impair autonomic cardiovascular regulation. Literature has suggested that supplemental oxygen enhances BRS significantly in patients with COPD mainly by decreasing sympathetic activity, pulmonary vasoconstriction and right ventricular afterload. ${ }^{11}$ Previous studies have shown that nocturnal hypoxemia is frequently present during sleep in patients with COPD with normal daytime $\mathrm{SpO}_{2} \cdot{ }^{41}$ However, clinical trials did not provide evidence on the long-time beneficial effects of NOT on survival and disease progression in patients with COPD and nocturnal hypoxemia. ${ }^{42-44}$ However, these studies might have been underpowered. A recent randomized controlled trial demonstrated that NOT did not have an effect on survival in patients with COPD who did not fill the prescription criteria for long-term oxygen therapy. ${ }^{22}$

There is some evidence on the beneficial effects of NOT on baroreflex sensitivity in COPD. Bartels et al studied the changes in BRS and cardiac indices in COPD in response to supplemental $\mathrm{O}_{2}$ or compressed air in a random order. ${ }^{11}$ They found that BRS significantly increased after $\mathrm{O}_{2}$ supplementation compared to the control group $(3.3 \pm 2.2 \mathrm{msec} / \mathrm{mmHg}$ vs $2.8 \pm 1.8 \mathrm{msec} / \mathrm{mm} \mathrm{Hg}$, $\mathrm{p}<0.05)$. According to these findings, blunted BRS at high altitude $(>5000 \mathrm{~m})$ was immediately ameliorated after inspiring oxygen. ${ }^{45}$ Mean BRS at low altitude was lower in our study population with COPD than in the general population (4.8vs. $6.8 \mathrm{~ms} / \mathrm{mmHg}$ ) and significantly fell to pathological values $<3 \mathrm{~ms} / \mathrm{mmHg}$ at altitude, while it remained unchanged when using NOT at altitude. The main trial of this study was previously published by Tan et al. ${ }^{26}$ In addition, Tan et al recently demonstrated favorable effects of the NOT in COPD during high altitude sojourn. The impaired well-being and night-time symptoms of patients with COPD at altitude were mitigated by NOT compared to the placebo therapy. ${ }^{26}$ Our analysis also suggests that NOT may have positive cardiovascular effects in moderate-to-severe COPD exposed to hypobaric hypoxia by improving BRS and decreasing BP. The beneficial effect of NOT might not only be due to correction of sustained hypoxemia but also due to avoiding pronounced intermittent hypoxemia as a consequence of 


\begin{tabular}{|c|c|c|c|c|}
\hline \multicolumn{2}{|l|}{ 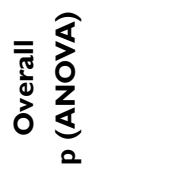 } & 资 훙 & $\bar{o}$ i & ఫ़ \\
\hline \multirow{2}{*}{ 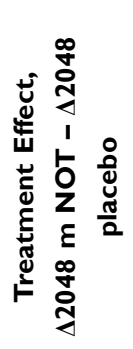 } & 2 & 능 స̄ & 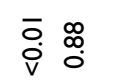 & 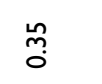 \\
\hline & 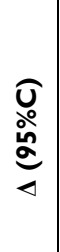 & 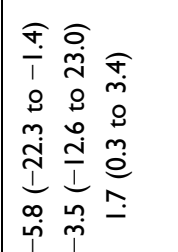 & 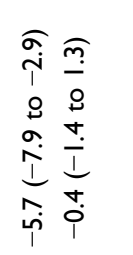 & $\begin{array}{l}\pi \\
0 \\
0 \\
0 \\
0 \\
i \\
\\
n \\
0\end{array}$ \\
\hline \multirow[b]{2}{*}{ 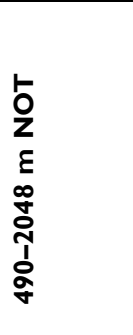 } & a & $\begin{array}{c}\infty \\
\infty \\
0 \\
0\end{array}$ ô & \begin{tabular}{ll}
\multirow{0}{*}{} & $\infty$ \\
0 & $\infty$ \\
0 & 0
\end{tabular} & ț \\
\hline & 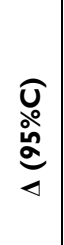 & 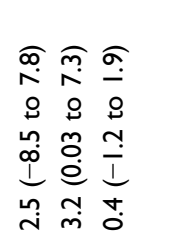 & 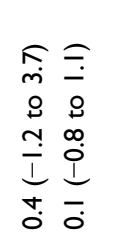 & $\begin{array}{l}\widehat{T} \\
0 \\
m \\
m \\
i \\
m \\
0\end{array}$ \\
\hline \multirow[b]{2}{*}{ 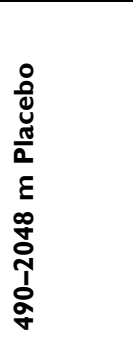 } & a & m ō & $\begin{array}{l}\overline{0} \\
\dot{0} \\
\dot{v}\end{array}$ & ثั \\
\hline & 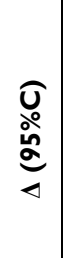 & 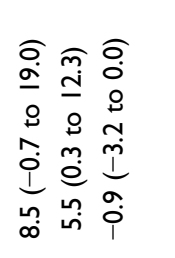 & 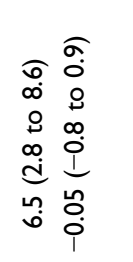 & 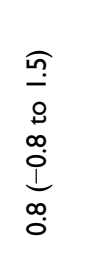 \\
\hline \multicolumn{2}{|l|}{ 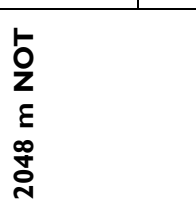 } & 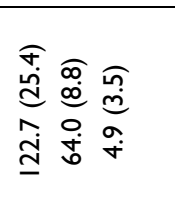 & 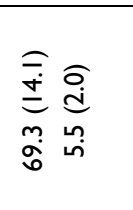 & $\begin{array}{l}\widehat{\widetilde{d}} \\
\stackrel{d}{\circ} \\
\dot{i}\end{array}$ \\
\hline \multicolumn{2}{|l|}{ 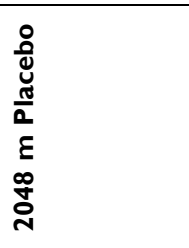 } & 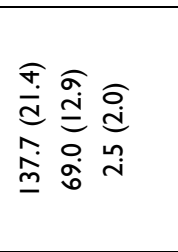 & 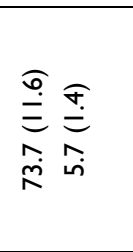 & 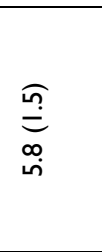 \\
\hline \multirow{2}{*}{\multicolumn{2}{|c|}{$\begin{array}{l}\varepsilon \\
\stackrel{g}{\circ} \\
\text { gे }\end{array}$}} & 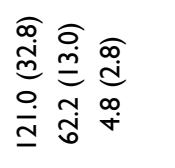 & 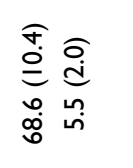 & $\begin{array}{l}\widehat{d} \\
\underset{\text { j̇ }}{n}\end{array}$ \\
\hline & & 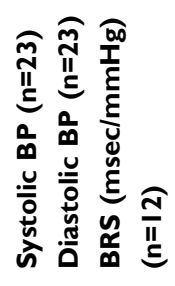 & 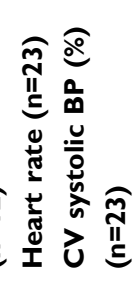 & 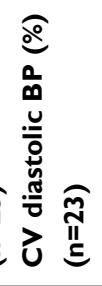 \\
\hline
\end{tabular}

altitude-induced periodic breathing/central sleep apnea. Several patients had some degree of obstructive respiratory events at low altitude and thus also intermittent hypoxemia (mean \pm SD oxygen desaturation index (ODI) at low altitude $9 \pm 12 / \mathrm{h}) .{ }^{26}$ However, the observed elevated ODI of $40 \pm 29 / \mathrm{h}$ at altitude on sham (room air) was mainly due to central sleep apnea at altitude.

A limitation of this study is its small sample size particularly for the assessment of BRS - that does not allow to reliably assess predictors of response to hypobaric hypoxia or NOT. However, such field studies are elaborative and patient's safety is of utmost importance (oxygen had to be given in several patients at altitude according to predefined safety criteria thus reducing the number of patients finishing the trial per protocol). However, for a trial focusing on cardiovascular physiology measures like BRS and BP, only a small sample size is required. One of the strengths of our study is the design as randomized-placebo-controlled, crossover study which allows to avoid many sources of bias. However, the fact that 9/32 patients could not be included in the per protocol analysis since they did not receive the allocated intervention at all three assessments due to altitude-related problems and thus had missing data on placebo at altitude may introduce a bias (survivor effect).

The effects of NOT on cardiovascular indices demonstrated in this randomized placebo-controlled interventional trial in moderate-to-severe COPD correspond to moderate altitude levels. These effects, which are relevant for patients (airplane traveling, work at altitude, resorts etc.), demonstrate the prevention of an altitude-induced clinically relevant increase in $\mathrm{BP}$ and a reduction in BRS. The extent of systolic blood pressure increase upon altitude exposure $(\Delta$ median $+8.5 \mathrm{mmHg}$ ) and the fall in BRS down to pathological values (from 4.8 down to 2.5 $\mathrm{msec} / \mathrm{mmHg}$, mean BRS in healthy people in this age group $8 \pm 3 \mathrm{msec} / \mathrm{mmHg}^{46}$ ) are of clinical relevance. A clinically relevant blood pressure increase at altitude was found, although almost half of patients were on at least one antihypertensive drug, mainly angiotensinconverting enzyme inhibitors or angiotensin receptor blockers. However, only one patient was on a betablocker, potentially influencing heart rate and BRS. Further studies are warranted to explore the cardiovascular effects of longer altitude sojourns and the effect of NOT in patients with COPD over a longer period of exposure to hypobaric hypoxia. 
A

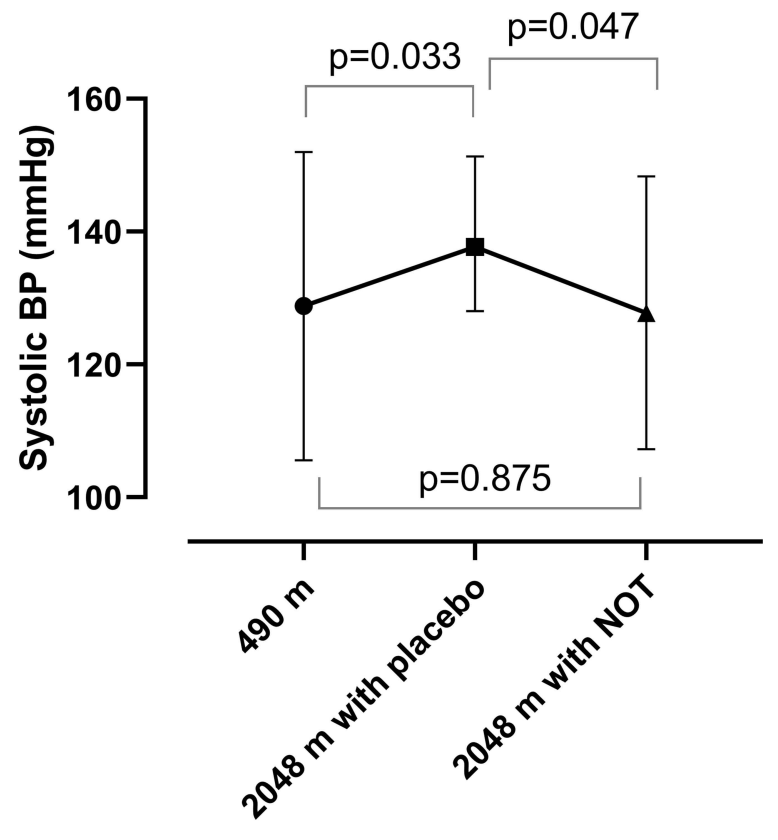

C

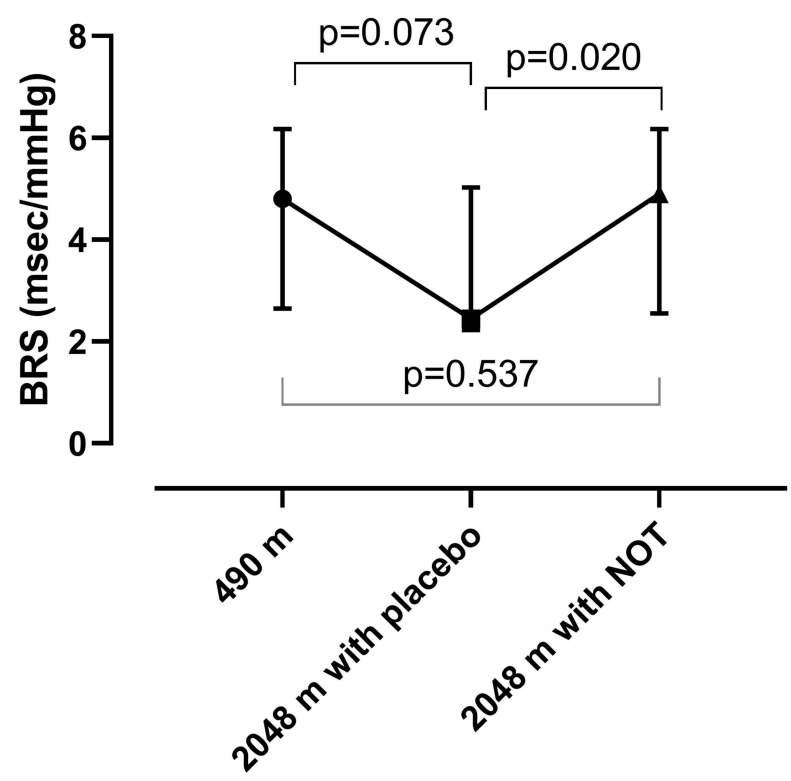

B
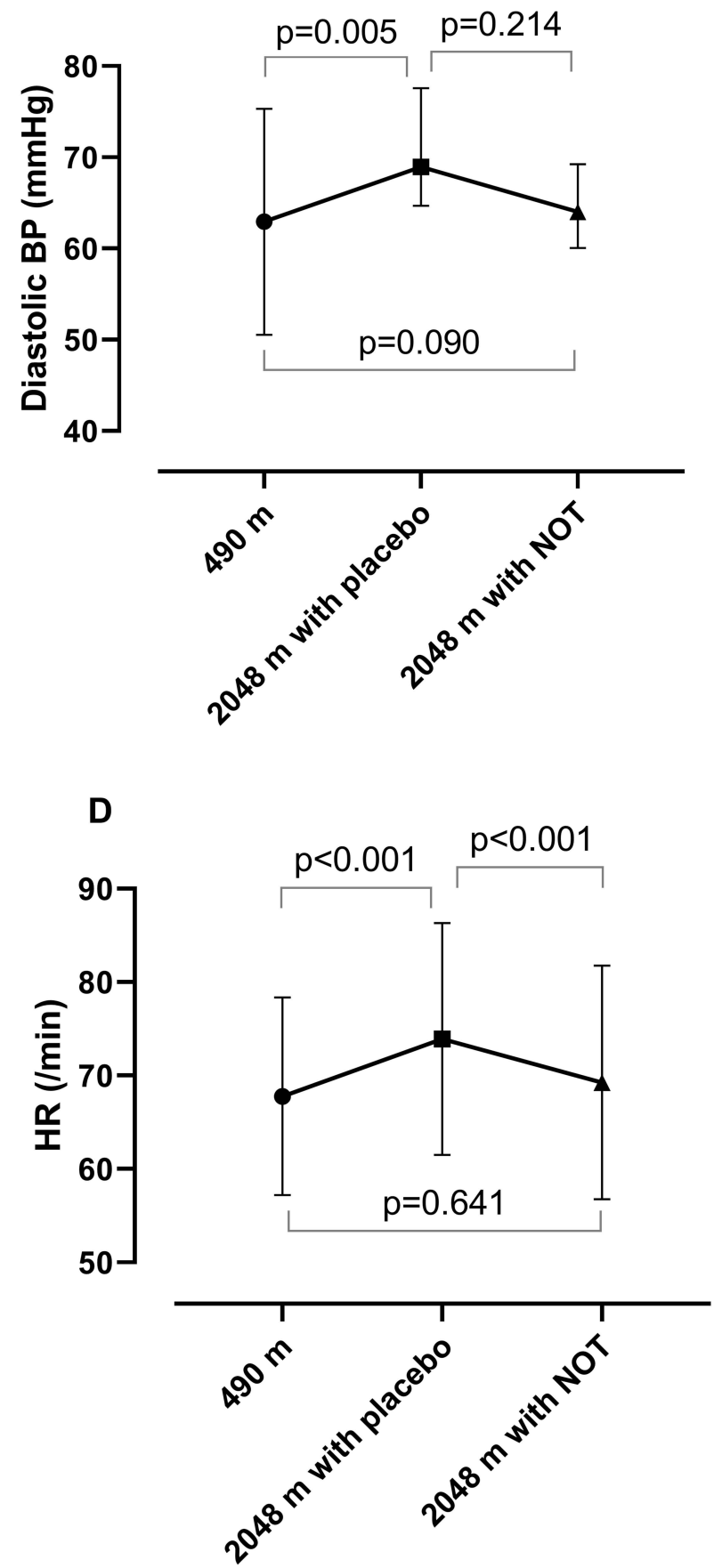

Figure 2 Systolic (A) and diastolic (B) blood pressure (BP), baroreflex sensitivity (BRS; (C) and heart rate (HR; (D) compared between the three assessments (median and IQR) - room air at low altitude $(490 \mathrm{~m})$, placebo (room air) at altitude (2048 m) and nocturnal oxygen therapy (NOT) at altitude (2048 m).

\section{Conclusion}

Acute exposure of patients with COPD to altitude is associated with a relevant increase in $\mathrm{BP}$ and a reduction in BRS that is ameliorated by the use of NOT. Therefore, and because NOT reduced altitude-related adverse health effects that required an intervention or descent, this study supports further research on cardiovascular and therapeutic effects of NOT in patients with COPD during prolonged altitude stays. 


\section{Abbreviations}

AHI, apnea-hypopnea-index; BP, blood pressure; BPV, blood pressure variability; BRS, baroreflex sensitivity; COPD, chronic obstructive pulmonary disease; NOT, nocturnal oxygen therapy; ODI, oxygen desaturation index.

\section{Data Sharing Statement}

Anonymized data can be requested by qualified researchers providing an approved proposal by contacting the corresponding author. Data will only be made available in case of approval of all authors.

\section{Ethical Statement}

The study was approved by the Ethics Committee of the Canton of Zurich (EK-2013-0088) and the main trial registered at ClinicalTrials.gov (registration number NCT02150590). All procedures were performed in accordance with the ethical standards of the institutional and national research committee and with the 1964 Helsinki declaration. Written informed consent was obtained from each participant.

\section{Acknowledgments}

The abstract of this paper was presented at the SSP/ SSTS-EFP Joint Annual Conference 2021 (Swiss Society of Pulmonology), 17-18 June, as a poster presentation. The poster's abstract was published in "Poster Abstracts" in Respiration 2021;100:644-750. Michael Furian and Esther I Schwarz are co-last authors of this study.

\section{Author Contributions}

All authors made a significant contribution to the work reported, whether that is in the conception, study design, execution, acquisition of data, analysis and interpretation, or in all these areas; took part in drafting, revising or critically reviewing the article; gave final approval of the version to be published; have agreed on the journal to which the article has been submitted; and agree to be accountable for all aspects of the work.

\section{Funding}

This work was supported by a Swiss National Science Foundation grant (32003B_143875) and a research grant of the LUNGE Zurich.

\section{Disclosure}

Prof. Dr. Silvia Ulrich reports grants from Swiss National Science Foundation, Swiss Lung League, Zurich Lung; grants, personal fees from Janssen/Actelion SA, Orpha Swiss; and personal fees from MSD SA, Switzerland, outside the submitted work. The authors declare that they do not have any financial or other conflict of interest to declare in relation with this work.

\section{References}

1. Adeloye D, Chua S, Lee C, et al. Global and regional estimates of COPD prevalence: systematic review and meta-analysis. $J$ Glob Health. 2015;5(2):020415. doi:10.7189/jogh.05.020415

2. Falk JA, Kadiev S, Criner GJ, Scharf SM, Minai OA, Diaz P. Cardiac disease in chronic obstructive pulmonary disease. Proc Am Thorac Soc. 2008;5(4):543-548. doi:10.1513/pats.200708-142ET

3. Hansen J, Sander M. Sympathetic neural overactivity in healthy humans after prolonged exposure to hypobaric hypoxia. $J$ Physiol. 2003;546(Pt 3):921-929. doi:10.1113/jphysiol.2002.031765

4. Chapleau MW. Determinants of baroreflex sensitivity in health and disease: from correlates to causality. Clin Auton Res. 2003;13 (5):310-313. doi:10.1007/s10286-003-0131-5

5. Cowley AW Jr., Liard JF, Guyton AC. Role of baroreceptor reflex in daily control of arterial blood pressure and other variables in dogs. Circ Res. 1973;32(5):564-576. doi:10.1161/01.RES.32.5.564

6. La Rovere MT, Pinna GD, Raczak G. Baroreflex sensitivity: measurement and clinical implications. Ann Noninvasive Electrocardiol. 2008;13(2):191-207. doi:10.1111/j.1542-474X.2008.00219.x

7. La Rovere MT, Bigger JT Jr., Marcus FI, Mortara A, Schwartz PJ. Baroreflex sensitivity and heart-rate variability in prediction of total cardiac mortality after myocardial infarction. ATRAMI (Autonomic Tone and Reflexes After Myocardial Infarction) investigators. Lancet. 1998;351(9101):478-484. doi:10.1016/S0140-6736(97)11144-8

8. Billman GE, Schwartz PJ, Stone HL. Baroreceptor reflex control of heart rate: a predictor of sudden cardiac death. Circulation. 1982;66 (4):874-880. doi:10.1161/01.CIR.66.4.874

9. Andreas S, Haarmann H, Klarner S, Hasenfuss G, Raupach T. Increased sympathetic nerve activity in COPD is associated with morbidity and mortality. Lung. 2014;192(2):235-241. doi:10.1007/ s00408-013-9544-7

10. Volterrani M, Scalvini S, Mazzuero G, et al. Decreased heart rate variability in patients with chronic obstructive pulmonary disease. Chest. 1994;106(5):1432-1437. doi:10.1378/chest.106.5.1432

11. Bartels MN, Gonzalez JM, Kim W, De Meersman RE. Oxygen supplementation and cardiac-autonomic modulation in COPD. Chest. 2000;118(3):691-696. doi:10.1378/chest.118.3.691

12. Chen W, Thomas J, Sadatsafavi M, FitzGerald JM. Risk of cardiovascular comorbidity in patients with chronic obstructive pulmonary disease: a systematic review and meta-analysis. Lancet Respir Med. 2015;3(8):631-639. doi:10.1016/S2213-2600(15)00241-6

13. Patakas D, Louridas G, Kakavelas E. Reduced baroreceptor sensitivity in patients with chronic obstructive pulmonary disease. Thorax. 1982;37(4):292-295. doi:10.1136/thx.37.4.292

14. Schwarz EI, Latshang TD, Furian M, et al. Blood pressure response to exposure to moderate altitude in patients with COPD. Int J Chron Obstruct Pulmon Dis. 2019;14:659-666. doi:10.2147/COPD. S194426

15. Graham WG, Houston CS. Short-term adaptation to moderate altitude. Patients with chronic obstructive pulmonary disease. JAMA. 1978;240(14):1491-1494. doi:10.1001/jama.1978.03290140033017

16. Stream JO, Luks AM, Grissom CK. Lung disease at high altitude. Expert Rev Respir Med. 2009;3(6):635-650. doi:10.1586/ers.09.51 
17. Furian M, Hartmann SE, Latshang TD, et al. Exercise performance of lowlanders with COPD at $2590 \mathrm{~m}$ : data from a randomized trial. Respiration. 2018;95(6):422-432. doi:10.1159/000486450

18. Furian M, Flueck D, Latshang TD, et al. Exercise performance and symptoms in lowlanders with COPD ascending to moderate altitude: randomized trial. Int J Chron Obstruct Pulmon Dis. 2018;13: 3529-3538. doi:10.2147/COPD.S173039

19. Somers VK, Mark AL, Abboud FM. Interaction of baroreceptor and chemoreceptor reflex control of sympathetic nerve activity in normal humans. J Clin Invest. 1991;87(6):1953-1957. doi:10.1172/ JCI115221

20. Parati G, Agostoni P, Basnyat B, et al. Clinical recommendations for high altitude exposure of individuals with pre-existing cardiovascular conditions: a joint statement by the European Society of Cardiology, the Council on Hypertension of the European Society of Cardiology, the European Society of Hypertension, the International Society of Mountain Medicine, the Italian Society of Hypertension and the Italian Society of Mountain Medicine. Eur Heart J. 2018;39 (17):1546-1554. doi:10.1093/eurheartj/ehx720

21. Hasler ED, Saxer S, Schneider SR, et al. Effect of breathing oxygen-enriched air on exercise performance in patients with Chronic Obstructive Pulmonary Disease: randomized, placebo-controlled, cross-over trial. Respiration. 2020;99(3):213-224. doi:10.1159/ 000505819

22. Lacasse Y, Series F, Corbeil F, et al. Randomized Trial of Nocturnal Oxygen in Chronic Obstructive Pulmonary Disease. $N$ Engl J Med. 2020;383(12):1129-1138. doi:10.1056/NEJMoa2013219

23. Cranston JM, Crockett AJ, Moss JR, Alpers JH. Domiciliary oxygen for chronic obstructive pulmonary disease. Cochrane Database Syst Rev. 2005;4:CD001744. doi:10.1002/14651858. CD001744.pub2

24. Nocturnal Oxygen Therapy Trial Group. Continuous or nocturnal oxygen therapy in hypoxemic chronic obstructive lung disease: a clinical trial. Ann Intern Med. 1980;93(3):391-398. doi:10.7326/ 0003-4819-93-3-391

25. Stuart H, Harris S, Tjh C. Long term domiciliary oxygen therapy in chronic hypoxic cor pulmonale complicating chronic bronchitis and emphysema. Report of the medical research council working party. Lancet. 1981;1(8222):681-686.

26. Tan L, Latshang TD, Aeschbacher SS, et al. Effect of nocturnal oxygen therapy on nocturnal hypoxemia and sleep apnea among patients with Chronic Obstructive Pulmonary Disease traveling to 2048 meters: a randomized clinical trial. JAMA Netw Open. 2020;3 (6):e207940. doi:10.1001/jamanetworkopen.2020.7940

27. Di Rienzo M, Castiglioni P, Parati G. The sequence technique revised: additional concepts on the assessment of spontaneous baroreflex function. Annu Int Conf IEEE Eng Med Biol Soc. 2010;2010:1703-1705. doi:10.1109/IEMBS.2010.5626844

28. Mozer MT, Holbein WW, Joyner MJ, Curry TB, Limberg JK. Reductions in carotid chemoreceptor activity with low-dose dopamine improves baroreflex control of heart rate during hypoxia in humans. Physiol Rep. 2016;4(13):e12859. doi:10.14814/phy2.12859

29. Querido JS, Wehrwein EA, Hart EC, Charkoudian N, Henderson WR, Sheel AW. Baroreflex control of muscle sympathetic nerve activity as a mechanism for persistent sympathoexcitation following acute hypoxia in humans. Am J Physiol Regul Integr Comp Physiol. 2011;301(6):R1779-R1785. doi:10.1152/ajpregu. 00182.2011

30. Bernardi L, Passino C, Spadacini G, et al. Cardiovascular autonomic modulation and activity of carotid baroreceptors at altitude. Clin Sci. 1998;95(5):565-573. doi:10.1042/cs0950565
31. Bourdillon N, Yazdani S, Subudhi AW, et al. AltitudeOmics: baroreflex sensitivity during acclimatization to $5260 \mathrm{~m}$. Front Physiol. 2018;9:767. doi:10.3389/fphys.2018.00767

32. Van De Borne P, Mezzetti S, Montano N, Narkiewicz K, Degaute JP, Somers VK. Hyperventilation alters arterial baroreflex control of heart rate and muscle sympathetic nerve activity. Am J Physiol Heart Circ Physiol. 2000;279(2):H536-541. doi:10.1152/ajpheart.2000.279.2.H536

33. Chaouat A, Naeije R, Weitzenblum E. Pulmonary hypertension in COPD. Eur Respir J. 2008;32(5):1371-1385. doi:10.1183/09031 936.00015608

34. Hubloue I, Rondelet B, Kerbaul F, et al. Endogenous angiotensin II in the regulation of hypoxic pulmonary vasoconstriction in anaesthetized dogs. Crit Care. 2004;8(4):R163-171. doi:10.1186/cc2860

35. Reid IA. Interactions between ANG II, sympathetic nervous system, and baroreceptor reflexes in regulation of blood pressure. Am J Physiol. 1992;262(6 Pt 1):E763-778. doi:10.1152/ajpendo.1992. 262.6.E763

36. Averill DB, Diz DI. Angiotensin peptides and baroreflex control of sympathetic outflow: pathways and mechanisms of the medulla oblongata. Brain Res Bull. 2000;51(2):119-128. doi:10.1016/S03619230(99)00237-3

37. Swierblewska E, Hering D, Kara T, et al. An independent relationship between muscle sympathetic nerve activity and pulse wave velocity in normal humans. $J$ Hypertens. 2010;28(5):979-984. doi:10.1097/ HJH.0b013e328336ed9a

38. McAllister DA, Maclay JD, Mills NL, et al. Arterial stiffness is independently associated with emphysema severity in patients with chronic obstructive pulmonary disease. Am J Respir Crit Care Med. 2007;176(12):1208-1214. doi:10.1164/rccm.200707-1080OC

39. Schillaci G, Bilo G, Pucci G, et al. Relationship between short-term blood pressure variability and large-artery stiffness in human hypertension: findings from 2 large databases. Hypertension. 2012;60 (2):369-377. doi:10.1161/HYPERTENSIONAHA.112.197491

40. McNicholas WT. Comorbid obstructive sleep apnoea and chronic obstructive pulmonary disease and the risk of cardiovascular disease. J Thorac Dis. 2018;10(Suppl 34):S4253-S4261. doi:10.21 037/jtd.2018.10.117

41. Lewis CA, Fergusson W, Eaton T, Zeng I, Kolbe J. Isolated nocturnal desaturation in COPD: prevalence and impact on quality of life and sleep. Thorax. 2009;64(2):133-138. doi:10.1136/thx.2007.088930

42. Fletcher EC, Luckett RA, Goodnight-White S, Miller CC, Qian W, Costarangos-Galarza C. A double-blind trial of nocturnal supplemental oxygen for sleep desaturation in patients with chronic obstructive pulmonary disease and a daytime $\mathrm{PaO} 2$ above $60 \mathrm{~mm} \mathrm{Hg}$. Am Rev Respir Dis. 1992;145(5):1070-1076. doi:10.1164/ajrccm/145.5.1070

43. Chaouat A, Weitzenblum E, Kessler R, et al. A randomized trial of nocturnal oxygen therapy in chronic obstructive pulmonary disease patients. Eur Respir J. 1999;14(5):1002. doi:10.1183/09031936.99. 14510029

44. Orth M, Walther JW, Yalzin S, et al. [Influence of nocturnal oxygen therapy on quality of life in patients with COPD and isolated sleep-related hypoxemia: a prospective, placebo-controlled cross-over trial]. Pneumologie. 2008;62(1):11-16. German. doi:10.1055/s-2007980129

45. Yazdani S, Bourdillon N, AltitudeOmics G, Vesin J-M AltitudeOmics: effect of exercise on baroreflex sensitivity at sea level and altitude; 2016.

46. Tank J, Baevski RM, Fender A, et al. Reference values of indices of spontaneous baroreceptor reflex sensitivity. Am J Hypertens. 2000;13 (3):268-275. doi:10.1016/S0895-7061(99)00172-7 


\section{Publish your work in this journal}

The International Journal of COPD is an international, peer-reviewed journal of therapeutics and pharmacology focusing on concise rapid reporting of clinical studies and reviews in COPD. Special focus is given to the pathophysiological processes underlying the disease, intervention programs, patient focused education, and self management protocols. This journal is indexed on PubMed Central, MedLine and CAS. The manuscript management system is completely online and includes a very quick and fair peer-review system, which is all easy to use. Visit http://www.dovepress.com/testimonials.php to read real quotes from published authors. 